ESAIM: COCV 19 (2013) 811-827

DOI: $10.1051 / \mathrm{cocv} / 2012034$
ESAIM: Control, Optimisation and Calculus of Variations

www.esaim-cocv.org

\title{
APPROXIMATE MAXIMUM PRINCIPLE FOR DISCRETE APPROXIMATIONS OF OPTIMAL CONTROL SYSTEMS WITH NONSMOOTH OBJECTIVES AND ENDPOINT CONSTRAINTS *
}

\author{
Boris S. Mordukhovich ${ }^{1}$ AND Ilya ShVARTSman ${ }^{2}$
}

\begin{abstract}
The paper studies discrete/finite-difference approximations of optimal control problems governed by continuous-time dynamical systems with endpoint constraints. Finite-difference systems, considered as parametric control problems with the decreasing step of discretization, occupy an intermediate position between continuous-time and discrete-time (with fixed steps) control processes and play a significant role in both qualitative and numerical aspects of optimal control. In this paper we derive an enhanced version of the Approximate Maximum Principle for finite-difference control systems, which is new even for problems with smooth endpoint constraints on trajectories and occurs to be the first result in the literature that holds for nonsmooth objectives and endpoint constraints. The results obtained establish necessary optimality conditions for constrained nonconvex finite-difference control systems and justify stability of the Pontryagin Maximum Principle for continuous-time systems under discrete approximations.
\end{abstract}

Mathematics Subject Classification. 49K15, 49M25, 49J52, 49J53, 93C55.

Received March 13, 2012. Revised August 17, 2012

Published online May 17, 2013.

\section{INTRODUCTION}

The classical result of optimal control theory is the Pontryagin Maximum Principle (PMP), which provides necessary optimality conditions for control systems governed by differential equations under various constraints; see the seminal book [8] and also, e.g., $[4,11]$ with the references therein for more recent developments and

\footnotetext{
Keywords and phrases. Discrete and continuous control systems, discrete approximations, constrained optimal control, maximum principles.

* Research of this author was partially supported by the USA National Science Foundation under grant DMS-1007132, by the Australian Research Council under grant DP-12092508, by the European Regional Development Fund (FEDER), and by the following Portuguese agencies: Foundation for Science and Technology, Operational Program for Competitiveness Factors, and Strategic Reference Framework under grant PTDC/MAT/111809/2009.

1 Department of Mathematics, Wayne State University Detroit, MI 48202, U.S.A. boris@math.wayne.edu

2 Department of Mathematics and Computer Science, Pennsylvania State University Harrisburg, Middletown, PA 17110, U.S.A. ius130psu.edu
} 
applications. For the basic optimal control problem written as

$$
(P)\left\{\begin{array}{l}
\text { minimize } \varphi_{0}\left(x\left(t_{1}\right)\right) \\
\text { subject to } \\
\dot{x}(t)=f(x(t), u(t), t) \text { a.e. } t \in T:=\left[t_{0}, t_{1}\right] \\
x\left(t_{0}\right)=x_{0} \in \mathbb{R}^{n} \\
u(t) \in U(t) \text { a.e. } t \in\left[t_{0}, t_{1}\right] \\
\varphi_{i}\left(x\left(t_{1}\right)\right) \leq 0, \quad i=1, \ldots, l \\
\varphi_{i}\left(x\left(t_{1}\right)\right)=0, \quad i=l+1, \ldots, l+q
\end{array}\right.
$$

the PMP asserts that every optimal control $\bar{u}(t)$ satisfies the maximum condition

$$
H(\bar{x}(t), p(t), \bar{u}(t), t)=\max _{u \in U(t)} H(\bar{x}(t), p(t), u, t) \text { a.e. } t \in T,
$$

along the corresponding trajectory $\bar{x}(t)$ of the primal system in $(P)$ and the solution $p(t)$ of the adjoint system

$$
\dot{p}(t)=-\nabla_{x} H(\bar{x}(t), p(t), \bar{u}(t), t) \text { a.e. } t \in T,
$$

when $f$ is smooth in $x$, with an appropriate transversality condition on $p\left(t_{1}\right)$ via gradients or subgradients of smooth or nonsmooth functions $\varphi_{i}$ at $\bar{x}\left(t_{1}\right)$. The Hamilton-Pontryagin function, or the unmaximized Hamiltonian, in (1.1) and (1.2) is given by

$$
H(x, p, u, t):=\langle p, f(x, u, t)\rangle, \quad p \in \mathbb{R}^{n} .
$$

This paper is devoted to the study of discrete approximations of the continuous-time control problem $(P)$ that are formalized as follows:

$$
\left(P_{N}\right)\left\{\begin{array}{l}
\text { minimize } \varphi_{0}\left(x_{N}\left(t_{1}\right)\right) \\
\text { subject to } \\
x_{N}\left(t+h_{N}\right)=x_{N}(t)+h_{N} f\left(x_{N}(t), u_{N}(t), t\right) \\
x_{N}\left(t_{0}\right)=x_{0} \in \mathbb{R}^{n} \\
u_{N}(t) \in U(t), \quad t \in T_{N}:=\left\{t_{0}, t_{0}+h_{N}, \ldots, t_{1}-h_{N}\right\} \\
\varphi_{i}\left(x_{N}\left(t_{1}\right)\right) \leq 0, \quad i=1, \ldots, l \\
\left|\varphi_{i}\left(x_{N}\left(t_{1}\right)\right)\right| \leq \delta>0, \quad i=l+1, \ldots, l+q \\
h_{N}:=\frac{t_{1}-t_{0}}{N}, \quad N \in \mathbb{N}:=\{1,2, \ldots\}
\end{array}\right.
$$

We treat $\left(P_{N}\right)$ as a sequence of discrete-time optimal control problems depending on the natural parameter $N=1,2, \ldots$ This sequence clearly arises from the Euler finite-difference replacement of the derivative in the differential equation of $(P)$ :

$$
\dot{x}(t) \approx \frac{x(t+h)-x(t)}{h} \text { as } h \rightarrow 0 .
$$

It is worth noting that we use here the uniform Euler scheme (1.4) just for simplicity; the approach and results obtained below can be carried over to other (including higher-order) approximation schemes.

Apart from the inevitable usage of discrete approximations in computer simulations and calculations of control systems with continuous time, there are deep interrelations between qualitative aspects of optimal control for continuous-time systems and their finite-difference approximations. On one hand, the method of discrete approximations has been very instrumental in deriving new necessary optimality conditions for various kinds of continuous-time control systems governed by differential equations and inclusions; see, e.g., $[3,4,10]$ and the references therein. On the other hand, it has been well recognized that the Discrete Maximum Principle (DMP), 
as an analog of the PMP with the exact maximum condition (1.1), does not generally hold for the discretetime problems $\left(P_{N}\right)$ with fixed steps $h_{N}$ if the admissible velocity sets $f(x, U(t), t)$ are not convex; see [4], Sections 6.4.1 and 6.5.23 for more details, examples, and historical background. This convexity assumption is rather restrictive, while the possibility to avoid it for continuous-time systems is due to a certain property of "hidden convexity" that is inherent in such systems and plays a crucial role (in one way or another) in any known proof of the PMP.

The failure of the DMP may create a potential instability of the PMP in modeling and numerical calculations of nonconvex continuous-time systems when using discrete approximations. Observe, however, that such stability would be justified by showing that merely an approximate counterpart of the PMP holds, with a certain perturbation of the maximum condition (1.1) diminishing together with the discretization step $h_{N} \downarrow 0$ as $N \rightarrow \infty$.

For smooth optimal control problems with no endpoint constraints, the first result of this type was obtained by Gabasov and Kirillova [1] under the name of "quasi-maximum principle" by using an analytic device exploiting the smooth and unconstrained nature of the family of parametric discrete problems considered therein.

Considering constrained control problems of type $\left(P_{N}\right)$ with smooth data required a completely different approach invoking the geometry of endpoint constraints under multineedle variations of optimal controls. It has been initiated by Mordukhovich [2], where a certain finite-difference counterpart of the hidden convexity property for sequences of constrained discrete-time systems was revealed and the first version of the Approximate Maximum Principle (AMP) for smooth control problems $\left(P_{N}\right)$ was established; the reader can find all the details and discussions in [4], Section 6.4 and commentaries therein.

The version of the AMP given in $[2,4]$ for smooth constrained problems $\left(P_{N}\right)$ can be formulated as follows. Let the pair $\left(\bar{u}_{N}, \bar{x}_{N}\right)$ be optimal to $\left(P_{N}\right)$ for each $N \in \mathbb{N}$ such that the control sequence $\left\{\bar{u}_{N}\right\}$ is proper (see $[2,4]$ and Section 3 below for the exact definition and discussions). Then for any $\varepsilon>0$ there exist multipliers $\lambda_{i N}$ as $i=0, \ldots, l+q$ normalized to

$$
\lambda_{0 N}^{2}+\ldots+\lambda_{l+q N}^{2}=1
$$

satisfying the sign and perturbed complementary slackness conditions

$$
\lambda_{i N} \geq 0 \text { for } i=0, \ldots, l \text { and }\left|\lambda_{i N} \varphi_{i}\left(\bar{x}_{N}\left(t_{1}\right)\right)\right|<\varepsilon \text { for } i=1, \ldots, l,
$$

and such that the approximate maximum condition

$$
H\left(\bar{x}_{N}(t), p_{N}\left(t+h_{N}\right), \bar{u}_{N}(t), t\right) \geq \max _{u \in U(t)} H\left(\bar{x}_{N}(t), p_{N}\left(t+h_{N}\right), u, t\right)-\varepsilon, \quad t \in T_{N},
$$

holds for all $N$ sufficiently large along the corresponding trajectory of the adjoint system

$$
p_{N}(t)=p_{N}\left(t+h_{N}\right)+h_{N} \frac{\partial H}{\partial x}\left(\bar{x}_{N}(t), p_{N}\left(t+h_{N}\right), \bar{u}_{N}(t), t\right), \quad t \in T_{N},
$$

with the endpoint/transversality condition

$$
-p_{N}\left(t_{1}\right)=\sum_{i=0}^{l+q} \lambda_{i N} \nabla \varphi_{i}\left(\bar{x}_{N}\left(t_{1}\right)\right) .
$$

The presented version of the AMP from $[2,4]$ plays actually the same role as the DMP for studying and solving discrete-time smooth optimal control problems with sufficiently small stepsizes without imposing the restrictive convexity assumption on the sets $f\left(\bar{x}_{N}(t), U(t), t\right), t \in T_{N}$. We refer the reader to, e.g., [4,7] and the bibliographies therein for more discussions and applications of the AMP to chemical engineering, periodic control, biotechnological and ecological processes, etc.

By analogy with the continuous-time problem $(P)$, it would be natural to expect that an extension of the AMP in form (1.5)-(1.9) could be obtained for problems $\left(P_{N}\right)$ with nonsmooth cost and constraint functions $\varphi_{i}$ 
via well-recognized subdifferentials of convex and nonsmooth analysis. But it has been surprisingly shown in [5] that this is not true even in the case of the simplest convex nondifferentiable cost function $\varphi_{0}(x)=|x-r|$ in the absence of any endpoint constraints on trajectories of linear one-dimensional control systems in $\left(P_{N}\right)$. This reveals a potential instability of the PMP under discrete approximations of problem $(P)$ with such a simple and standard type of nonsmoothness when the AMP in form (1.5)-(1.9) is applied. Note that nonsmooth control problems are not only highly challenging theoretically, but are important for various practical applications to engineering design, finance, environmental science, economics, etc.; see, e.g., $[4,10,11]$ and the references therein.

The main goal of this paper is to develop a new version of the AMP that holds for problems $\left(P_{N}\right)$ with nonsmooth objectives and endpoint inequality constraints represented as sums of convex and smooth (in fact, strictly differentiable) functions, while equality constraint functions remain smooth. In this version of the AMP, which is new even for problems $\left(P_{N}\right)$ with all functions $\varphi_{i}$ being smooth, we keep the approximate maximum condition (1.7) together with the adjoint system (1.8) as well as the nontriviality and sign conditions on $\lambda_{i N}$ in (1.5) and (1.6), respectively. What is modified is that we replace the perturbed complementarity slackness condition in (1.6) by its exact counterpart

$$
\lambda_{i N} \varphi_{i}(\bar{x})=0 \text { for all } i=1, \ldots, l
$$

and replace the transversality condition (1.9) by its subdifferential analog

$$
-p_{N}\left(t_{1}\right) \in \sum_{i=0}^{l} \lambda_{i N} \partial \varphi_{i}(\bar{x})+\sum_{i=l+1}^{l+q} \lambda_{i N} \nabla \varphi_{i}(\bar{x}),
$$

where $\bar{x}$ in is a limiting point of the sequence $\left\{\bar{x}_{N}\left(t_{1}\right)\right\}$, and where $\partial \varphi_{i}(\bar{x})$ in (1.10) stands for the sum of the subdifferential of convex analysis and the classical gradient at $\bar{x}$ of the corresponding terms in the aforementioned representations of $\varphi_{i}$ as $i=0, \ldots, l$.

The rest of the paper is organized as follows. In Section 2 we recall some definitions and preliminaries needed for formulations and proofs of the main results. Section 3 contains the exact formulations and discussions of the assumptions and the statement of the new version of the AMP developed in the paper for the sequence of problems $\left(P_{N}\right)$. We also discuss the corresponding results for discrete approximation problems of type $\left(P_{N}\right)$ with relaxations of endpoint constraints. Section 4 presents the proof of the main theorem split into several lemmas, which are of their own interest. In the concluding Section 5 we discuss endpoint constraints of the equality type and give an example showing that the new version of the AMP does not hold for such problems without equality constraint relaxations.

Throughout the paper we use the standard notation from variational analysis, optimization, and optimal control; see, e.g., the books $[4,11]$.

\section{PRELIMinaRies}

For the reader's convenience, in this section we present several definitions and well-known facts widely used in what follows.

Recall that $\varphi: \mathbb{R}^{n} \rightarrow \mathbb{R}$ is strictly differentiable at $\bar{x}$ if there is a vector $\nabla \varphi(\bar{x})$ such that

$$
\lim _{x_{1}, x_{2} \rightarrow \bar{x}} \frac{\varphi\left(x_{1}\right)-\varphi\left(x_{2}\right)-\left\langle\nabla \varphi(\bar{x}), x_{1}-x_{2}\right\rangle}{\left\|x_{1}-x_{2}\right\|}=0 .
$$

Strict differentiability at a point is a stronger property that just (Fréchet) differentiability, but weaker than continuous differentiability $\left(\right.$ i.e., $C^{1}$ ) around $\bar{x}$. A classical example of a function that is differentiable while not strictly differentiable at $\bar{x}=0$ is

$$
\varphi(x):= \begin{cases}x^{2} \sin \frac{1}{x}, & x \neq 0 \\ 0, & x=0 .\end{cases}
$$


Note that just differentiability of the cost and constraint functions is needed for the PMP to hold in the continuous-time problem $(P)$ (see [4], Th. 6.37) in contrast to the corresponding strict differentiability assumptions required for the validity of the AMP in the previous and new forms; $c f$. Theorem 6.59 and Example 6.55 in [4] (the latter is taken from [5], Ex. 2.3) and Section 3 below.

If $\varphi: \mathbb{R}^{n} \rightarrow \overline{\mathbb{R}}:=\mathbb{R} \cup\{\infty\}$ is a convex function finite at $\bar{x}$, its subdifferential at this point in the sense of convex analysis is defined by

$$
\partial \varphi(\bar{x}):=\left\{v \in \mathbb{R}^{n} \mid\langle v, x-\bar{x}\rangle \leq \varphi(x)-\varphi(\bar{x}) \text { for all } x \in \mathbb{R}^{n}\right\} .
$$

It is well known (see, e.g., [9]) that $\partial \varphi(\bar{x}) \neq \emptyset$ for any $\bar{x} \in \operatorname{dom} \varphi:=\left\{x \in \mathbb{R}^{n} \mid \varphi(x)<\infty\right\}$, that $\varphi$ is locally Lipschitz continuous on the interior of $\operatorname{dom} \varphi$ and that

$$
\|v\| \leq \ell \text { for all } v \in \partial \varphi(\bar{x})
$$

where $\ell>0$ is the local Lipschitz constant of $\varphi$ at $\bar{x}$. Note also that the subdifferential $\partial \varphi(\bar{x})$ can be equivalently represented as

$$
\partial \varphi(\bar{x})=\left\{v \in \mathbb{R}^{n} \mid\langle v, z\rangle \leq \varphi^{\prime}(\bar{x}, z) \text { for all } z \in \mathbb{R}^{n}\right\}
$$

via the directional derivative $\varphi^{\prime}(\bar{x} ; z)$ of $\varphi$ at $\bar{x}$ in the direction $z$ defined by

$$
\varphi^{\prime}(\bar{x} ; z):=\lim _{\alpha \downarrow 0, w \rightarrow z} \frac{\varphi(\bar{x}+\alpha w)-\varphi(\bar{x})}{\alpha},
$$

which, for Lipschitz functions, is equivalent to

$$
\varphi^{\prime}(\bar{x} ; z):=\lim _{\alpha \downarrow 0} \frac{\varphi(\bar{x}+\alpha z)-\varphi(\bar{x})}{\alpha} .
$$

This implies, in turn, the directional derivative representation

$$
\varphi^{\prime}(\bar{x} ; z)=\sup _{v \in \partial \varphi(\bar{x})}\langle v, z\rangle .
$$

Given a convex set $\Omega \subset \mathbb{R}^{n}$ and a point $\bar{x} \in \Omega$, the normal cone to $\Omega$ at $\bar{x}$ is defined by

$$
\mathcal{N}(\bar{x} ; \Omega):=\left\{v \in \mathbb{R}^{n} \mid\langle v, x-\bar{x}\rangle \leq 0 \text { for all } x \in \Omega\right\} .
$$

The following results of convex analysis are widely used in the paper; see [9] for more details.

Subdifferential sum rule. Let $\varphi, \psi$ be convex functions on $\mathbb{R}^{n}$ such that $\psi$ is differentiable at $\bar{x} \in \operatorname{dom} \varphi$. Then we have

$$
\partial(\psi+\varphi)(\bar{x})=\nabla \psi(\bar{x})+\partial \varphi(\bar{x})
$$

Note that this sum rule is a simple particular case of the Moreau-Rockafellar Theorem, which is a fundamental result of convex analysis.

Carathéodory theorem. If a point $x \in \mathbb{R}^{n}$ lies in the convex hull of a set $P$, there is a subset $\widetilde{P} \subset P$ consisting of at most $n+1$ points such that $x$ belongs to the convex hull of $\widetilde{P}$.

Separation theorem. Let $C, D \subset \mathbb{R}^{n}$ be convex sets such that int $C \neq \emptyset$ and $\operatorname{int} C \cap D=\emptyset$. Then there exists a nonzero vector $v \in \mathbb{R}^{n}$ such that

$$
\left\langle v, x_{1}\right\rangle \leq\left\langle v, x_{2}\right\rangle \text { for all } x_{1} \in C, x_{2} \in D .
$$




\section{The MAIN RESUlt AND Discussions}

Throughout the paper we assume that the control sets $U(t)$ in $\left(P_{N}\right)$ are compact subsets of a metric space $(\mathcal{U}, d)$ and that the set-valued mapping $U:\left[t_{0}, t_{1}\right] \rightrightarrows \mathcal{U}$ is continuous with respect to the Hausdorff distance. Recall $[2,4]$ that a sequence of discrete-time control $\left\{u_{n}\right\}$ in $\left(P_{N}\right)$ is proper if for every sequence of mesh points

$$
\tau_{\theta(N)}:=t_{0}+\theta(N) h_{N} \in T_{N} \text { with } \theta(N)=0, \ldots, N-1 \text { and } \tau_{\theta(N)} \rightarrow t \in\left[t_{0}, t_{1}\right] \text { as } N \rightarrow \infty
$$

we have the following relationships:

$$
\text { either } d\left(u_{N}\left(\tau_{\theta(N)}\right), u_{N}\left(\tau_{\theta(N)+s}\right)\right) \rightarrow 0, \text { or } d\left(u_{N}\left(\tau_{\theta(N)}\right), u_{N}\left(\tau_{\theta(N)-s}\right) \rightarrow 0,\right.
$$

or both hold as $N \rightarrow \infty$ with any natural constant $s$.

Note that this property postulates at least one-sided continuity in the limiting sense of the control sequence in $\left(P_{N}\right)$ at every point and is a discrete-time analog of Lebesgue points in continuous time, which comprise a set of full measure. Thus is not required for continuous-time control systems; see [4] for more details. However, this property occurs to be essential for the validity of the AMP even for one-dimensional linear systems with linear cost and constraint functions; see [5], Example 2.1 and [4], Theorem 6.60.

Theorem 3.1 (Approximate maximum principle for nonsmooth control problems). Let the pair $\left(\bar{u}_{N}, \bar{x}_{N}\right)$ be optimal to problems $\left(P_{N}\right)$ as $N \in \mathbb{N}$, the sequence $\left\{\bar{x}_{N}\left(t_{1}\right)\right\}$ is uniformly bounded, and let $\bar{x}$ be a limiting point of this sequence. Assume that:

(a) The function $f$ is continuous with respect to its variables and continuously differentiable with respect to $x$ in a tube containing the optimal trajectories $\bar{x}_{N}(t)$ for all large $N$.

(b) The cost function $\varphi_{0}$ and the inequality constraint functions $\varphi_{i}$ admit the representations

$$
\varphi_{i}(x)=\psi_{i}(x)+\vartheta_{i}(x) \text { for } i=0, \ldots, l,
$$

where each $\psi_{i}$ is strictly differentiable at $\bar{x}$, and where each $\vartheta_{i}$ is convex.

(c) The equality constraint functions $\varphi_{i}$ as $i=l+1, \ldots, l+q$ are strictly differentiable at $\bar{x}$.

(d) The sequence of optimal controls $\left\{\bar{u}_{N}\right\}$ is proper.

Then for any $\varepsilon>0$ there exist a natural number $N_{\varepsilon}$ and multipliers $\lambda_{i N} \in \mathbb{R}$ as $i=0, \ldots, l+q$ such that for all $N \geq N_{\varepsilon}$ the following hold:

- The Sign and NONTRIVIALITY CONDITIONS

$$
\lambda_{i N} \geq 0 \quad \text { as } i=0, \ldots, l \quad \text { and } \quad \sum_{i=0}^{l} \lambda_{i N}^{2}=1 ;
$$

- The COMPlementary SLACKNess CONDitions

$$
\lambda_{i N} \varphi_{i}(\bar{x})=0 \text { as } i=1, \ldots, l \text {; }
$$

- The approximate maximum Condition (1.7), where $p_{N}(t)$ as $t \in T_{N} \cup\left\{t_{1}\right\}$ is the solution of the adjoint system (1.8) satisfying

- The ENDPOINT/TRANSVERSALITY CONDITION

$$
-p_{N}\left(t_{1}\right) \in \sum_{i=0}^{l} \lambda_{i N}\left(\nabla \psi_{i}(\bar{x})+\partial \vartheta_{i}(\bar{x})\right)+\sum_{i=l+1}^{l+q} \lambda_{i N} \nabla \varphi_{i}(\bar{x}) .
$$


Note that the new AMP of Theorem 3.1 is an appropriate finite-difference counterpart of the corresponding nonsmooth PMP for continuous-time system $(P)$; see $[4,11]$. Also, we would like to emphasize that the new result differs from the previous version of the AMP for smooth problems $\left(P_{N}\right)$ discussed in Section 1 in the following two major aspects:

(i) In contrast to (1.6), the complementary slackness conditions (3.2) are exact and are evaluated at the limiting point $\bar{x}$ rather than at $\bar{x}_{N}\left(t_{1}\right)$.

(ii) In contrast to (1.9), the right-hand side of the transversality condition (3.3) is evaluated at the limiting point $\bar{x}$ rather than at $\bar{x}_{N}\left(t_{1}\right)$.

Note that the result of Theorem 3.1 was announced in [6] for the case of convex cost and endpoint constraint functions $\varphi_{i}, i=0, \ldots, l$.

Remark 3.2 (AMP under constraint perturbations). It follows from the proof of Theorem 3.1 given in the next section that it is possible to derive the AMP in the new form of this theorem under arbitrary perturbations of the inequality constraints in $\left(P_{N}\right)$ :

$$
\varphi_{i}\left(x_{N}\left(t_{1}\right)\right) \leq \gamma_{i N} \text { with } \gamma_{i N} \rightarrow 0 \text { as } N \rightarrow \infty, \quad i=1, \ldots, l,
$$

keeping all other assumptions in place. Perturbations of this type were considered in [2, 4], where the AMP was derived in form (1.5)-(1.9) in the case of smooth cost and constraint functions. Note that the previous form of the AMP was also obtained in [2,4] for perturbations of the equality constraints

$$
\left|\varphi_{i N}\left(x_{N}\left(t_{1}\right)\right)\right| \leq \delta_{i N} \text { with } \limsup _{N \rightarrow \infty} \frac{h_{N}}{\delta_{i N}}=0 \text { for all } i=l+1, \ldots, l+q .
$$

The question about the validity of the AMP in the new form of Theorem 3.1 under the general consistent equality constraint perturbations as in (3.4) remains open. On the other hand, in Section 5 we present a counterexample to this statement for $\delta_{i N} \equiv 0$ as well as for the case of too quickly vanishing (inconsistent) perturbations $\delta_{i N}$ in (3.4).

\section{Proof of the theorem}

Suppose first that Theorem 3.1 is valid in the case $q=0$, that is, in the absence of the relaxed equality constraints $\left|\varphi_{i}\left(x_{N}\left(t_{1}\right)\right)\right| \leq \delta, i=l+1, \ldots, l+q$. Let us show that Theorem 3.1 is then valid when $q>0$.

Indeed, each of these constraints can be written as the two inequalities:

$$
\varphi_{i}\left(x_{N}\left(t_{1}\right)\right) \leq \delta \text { and }-\varphi_{i}\left(x_{N}\left(t_{1}\right)\right) \leq \delta \text { for } i=l+1, \ldots, l+q .
$$

Fix an index $i \in\{l+1, \ldots, l+q\}$ in (4.1). Due to our assumption on validity of Theorem 3.1 in the absence of relaxed equality constraints and applying (3.2), we can find $\lambda_{i N}^{+} \geq 0$ and $\lambda_{i N}^{-} \geq 0$ such that

$$
\lambda_{i N}^{+} \varphi_{i}(\bar{x})=\lambda_{i N}^{+} \delta \text { and }-\lambda_{i N}^{-} \varphi_{i}(\bar{x})=\lambda_{i N}^{-} \delta .
$$

Assuming for definiteness that $\lambda_{i N}^{+} \neq 0$, we get from the first equality in (4.2) that $\varphi_{i}(\bar{x})=\delta$ and thus $-2 \delta \lambda_{i N}^{-}=0$ from the second one. Since $\delta \neq 0$, it gives that $\lambda_{i N}^{-}=0$. Denoting finally $\lambda_{i N}:=\lambda_{i N}^{+}-\lambda_{i N}^{-}$for the chosen index $i \in\{l+1, \ldots, l+q\}$ in the last term on (3.3), we arrive at all the conclusions of Theorem 3.1, as formulated.

Therefore, in what follows we prove Theorem 3.1 in the case $q=0$ breaking down the proof into a series of lemmas.

The first lemma presents a simple subdifferential property of convex continuous functions needed in what follows. 
Lemma 4.1 (subdifferential property of convex functions). Let $\varphi: \mathbb{R}^{n} \rightarrow \mathbb{R}$ be a convex continuous function, and let $y_{k} \rightarrow 0$ as $k \rightarrow \infty$ for some sequence $\left\{y_{k}\right\} \subset \mathbb{R}^{n}$. Then given $\bar{x} \in \mathbb{R}^{n}$, there exists a subgradient $v \in \partial \varphi(\bar{x})$ such that

$$
\varphi\left(\bar{x}+y_{k}\right)-\varphi(\bar{x})=\left\langle v, y_{k}\right\rangle+o\left(\left\|y_{k}\right\|\right) \quad \text { as } k \rightarrow \infty
$$

along a subsequence of $\left\{y_{k}\right\}$, without relabeling.

Proof. Since the assertion of the lemma is obvious when $y_{k} \equiv 0$, we suppose without loss of generality that $\left\{y_{k}\right\}$ contains a nonzero subsequence. Let $y_{k} /\left\|y_{k}\right\| \rightarrow z$ along such a subsequence. Due to (2.2) we have the representation

$$
\varphi\left(\bar{x}+y_{k}\right)-\varphi(\bar{x})=\varphi\left(\bar{x}+\frac{y_{k}}{\left\|y_{k}\right\|}\left\|y_{k}\right\|\right)-\varphi(\bar{x})=\varphi\left(\bar{x}+\left(z+w_{k}\right)\left\|y_{k}\right\|\right)-\varphi(\bar{x})=\varphi^{\prime}(\bar{x} ; z)\left\|y_{k}\right\|+o\left(\left\|y_{k}\right\|\right),
$$

where $w_{k} \rightarrow 0$ as $k \rightarrow \infty$. Taking into account the directional derivative representation (2.3) as well as the nonemptiness and compactness of the subdifferential of convex and continuous (hence Lipschitz continuous) functions, we find a subgradient $v \in \partial \varphi(\bar{x})$ such that

$$
\varphi^{\prime}(\bar{x} ; z)=\langle v, z\rangle .
$$

This implies, therefore, the relationships

$$
\varphi\left(\bar{x}+y_{k}\right)-\varphi(\bar{x})=\langle v, z\rangle\left\|y_{k}\right\|+o\left(\left\|y_{k}\right\|\right)=\left\langle v, \frac{y_{k}}{\left\|y_{k}\right\|}\right\rangle\left\|y_{k}\right\|+o\left(\left\|y_{k}\right\|\right)=\left\langle v, y_{k}\right\rangle+o\left(\left\|y_{k}\right\|\right),
$$

which completes the proof of the lemma.

Next we prove a technical lemma, which plays a significant role in the proof of the main result.

Lemma 4.2 (limiting property of convex functions). Let $\varphi: \mathbb{R}^{n} \rightarrow \mathbb{R}$ be a convex continuous function, $\left\{x_{k}\right\},\left\{y_{k}\right\} \subset \mathbb{R}^{n}$ and $\left\{\widetilde{c}_{k}\right\} \subset \mathbb{R}$ be such sequences that $x_{k} \rightarrow \bar{x}, y_{k} \rightarrow 0$, and $\widetilde{c}_{k}=o\left(\left\|y_{k}\right\|\right)$ as $k \rightarrow \infty$, and let

$$
\varphi\left(x_{k}+y_{k}\right) \geq \varphi\left(x_{k}\right)+\widetilde{c}_{k} \quad \text { for all } k \in \mathbb{N} .
$$

Then there exists a sequence $\left\{c_{k}\right\} \subset \mathbb{R}$ such that $c_{k}=o\left(\left\|y_{k}\right\|\right)$ as $k \rightarrow \infty$ and

$$
\varphi\left(\bar{x}+y_{k}\right) \geq \varphi(\bar{x})+c_{k} \quad \text { for all } k \in \mathbb{N} .
$$

Proof. Observe first that by modifying the sequence $\left\{\widetilde{c}_{k}\right\}$ if necessary, we can reduce (4.3) to the strict inequality

$$
\varphi\left(x_{k}+y_{k}\right)>\varphi\left(x_{k}\right)+\widetilde{c}_{k} \text { for all } k \in \mathbb{N},
$$

which can be restated in the equivalent form $\left(x_{k}, \varphi\left(x_{k}\right)\right)+\left(y_{k}, \widetilde{c}_{k}\right) \notin$ epi $\varphi$. Denote

$$
\alpha_{k}:=\max \left\{\alpha \geq 0 \mid\left(x_{k}, \varphi\left(x_{k}\right)\right)+\alpha\left(y_{k}, \widetilde{c}_{k}\right) \in \operatorname{epi} \varphi\right\}
$$

observing that the maximum is reached in (4.5) for some $\alpha_{k} \in[0,1)$ due to closedness and convexity of the epigraph epi $\varphi$ of $\varphi$.

Suppose without loss of generality that $\left\{y_{k}\right\}$ contains a nonzero subsequence, and let $A$ be the set of the limiting points of $\left\{y_{k} /\left\|y_{k}\right\|\right\}$, i.e.,

$$
A:=\left\{z \in \mathbb{R}^{n} \mid z=\lim _{k \rightarrow \infty} \frac{y_{k}}{\left\|y_{k}\right\|} \text { along a subsequence of } k \in \mathbb{N}\right\} .
$$

Considering further the union set

$$
B:=\bigcup_{\beta>0}(\bar{x}+\beta A),
$$


we claim the relationship

$$
(B, f(\bar{x})) \cap \operatorname{int}(\text { epi } f)=\emptyset .
$$

Indeed, assume otherwise that there exist $\widetilde{\beta}>0$ and $z \in A$ such that

$$
(\bar{x}+\widetilde{\beta} z, \varphi(\bar{x})) \in \operatorname{int}(\operatorname{epi} \varphi) .
$$

This implies that for any sequence converging to $(\bar{x}+\widetilde{\beta} z, \varphi(\bar{x}))$ all its terms belong to the epigraph of $\varphi$ starting with a certain number. In particular, there exists a subsequence of $y_{k}$ (without relabeling) such that

$$
\left(x_{k}+\alpha_{k} y_{k}+\widetilde{\beta} \frac{y_{k}}{\left\|y_{k}\right\|}, \varphi\left(x_{k}\right)+\alpha_{k} \widetilde{c}_{k}+\widetilde{\beta} \frac{\widetilde{c}_{k}}{\left\|y_{k}\right\|}\right) \in \operatorname{epi} \varphi
$$

for sufficiently large $k \in \mathbb{N}$; this can be written as

$$
\left(x_{k}+\left(\alpha_{k}+\frac{\widetilde{\beta}}{\left\|y_{k}\right\|}\right) y_{k}, \varphi\left(x_{k}\right)+\left(\alpha_{k}+\frac{\widetilde{\beta}}{\left\|y_{k}\right\|}\right) \widetilde{c}_{k}\right) \in \operatorname{epi} \varphi .
$$

It contradicts the definition of $\alpha_{k}$ in (4.5) and thus justifies the empty intersection in (4.6).

Furthermore, we have from the construction of the set $A$ that

$$
\bar{x}+y_{k}=\bar{x}+\left\|y_{k}\right\|\left(\frac{y_{k}}{\left\|y_{k}\right\|}\right) \in \bar{x}+\left\|y_{k}\right\| A+z_{k}
$$

with some sequence $\left\{z_{k}\right\} \subset \mathbb{R}^{n}$ of order $o\left(\left\|y_{k}\right\|\right)$. This implies that

$$
\left(\bar{x}+y_{k}-z_{k}, \varphi(\bar{x})\right) \in\left(\bar{x}+\left\|y_{k}\right\| A, \varphi(\bar{x})\right) \subset(B, f(\bar{x}))
$$

and hence $\left(\bar{x}+y_{k}-z_{k}, \varphi(\bar{x})\right) \notin \operatorname{int}(\operatorname{epi} \varphi)$ due to (4.6), which means that

$$
\varphi\left(\bar{x}+y_{k}-z_{k}\right) \geq \varphi(\bar{x}) .
$$

Due to Lipschitz continuity of $\varphi$ around $\bar{x}$ with some constant $\ell$ we get

$$
\varphi\left(\bar{x}+y_{k}-z_{k}\right) \leq \varphi\left(\bar{x}+y_{k}\right)+\ell\left\|z_{k}\right\| .
$$

Setting finally $c_{k}:=-\ell\left\|z_{k}\right\|$ and combining (4.7) with (4.8) ensures the validity of (4.4) and thus completes the proof of the lemma.

Note that the assertion of Lemma 4.2 does not generally hold for nonconvex nonsmooth functions. A simple example is provided by $\varphi(x)=-|x|$ with the sequences $x_{k}=-1 / k, y_{k}=1 / k$, and $\widetilde{c}_{k}=0$. Then we have (4.3) along these sequences while (4.4) reduces to $-1 / k \geq 0+c_{k}$, which does not hold whenever $c_{k}=o(1 / k)$ as $k \rightarrow \infty$.

Having in hand the technical lemmas established above, we are now ready to proceed with the proof of the AMP in Theorem 3.1. First we recall a significant property of finite-difference control systems related to needle variations of optimal controls.

Let $\left(\bar{x}_{N}, \bar{u}_{N}\right)$ be optimal process to problems $\left(P_{N}\right)$ for $N \in \mathbb{N}$. Fix a natural number $r \in\{1, \ldots, N-1\}$, mesh points $\tau_{j}(N) \in T_{N}$, and control impulses $v_{j}(N) \in U\left(\tau_{j}(N)\right)$ and then define an $r$-needle variation of the optimal control $\bar{u}_{N}$ by

$$
u_{N}^{r}(t):= \begin{cases}v_{j}(N), & t=\tau_{j}(N), \\ \bar{u}_{N}(t), & t \in T_{N}, t \neq \tau_{j}(N), j=1, \ldots, r .\end{cases}
$$


The case of $r=1$ in (4.9) defines a single needle variation of $\bar{u}$, and we omit the index $r=1$ in this case. The corresponding trajectory increments are denoted by $\Delta^{r} x_{N}(t):=x_{N}^{r}(t)-\bar{x}_{N}(t)$ and $\Delta x_{N}(t):=x_{N}(t)-\bar{x}_{N}(t)$ when $r=1$. Next we pick natural numbers $p$ and $m_{j}$ as $j=1, \ldots, p$ independent of $N$ and consider the integer combination

$$
\Delta_{N}\left(p, m_{j}\right):=\sum_{j=1}^{p} m_{j} \Delta_{j} x_{N}\left(t_{1}\right)
$$

of the endpoint trajectory increments $\Delta_{j} x_{N}\left(t_{1}\right)$ generated by $p$ single needle control variations $u_{N j}(t)$. The following lemma taken from [4], Lemma 6.62 shows that any single needle integer combination of type (4.10) can be approximated up to a small quantity of order $o\left(h_{N}\right)$ by an endpoint trajectory increment generated by a multineedle control variation.

Lemma 4.3 (needle variations). Let the reference sequence of optimal controls $\left\{\bar{u}_{N}\right\}$ to $\left(P_{N}\right)$ be proper, and let $\Delta_{N}\left(p, m_{j}\right)$ be the integer combination (4.10) generated by single needle control variations $u_{N j}(t), j=1, \ldots, p$. Then there exists an r-needle control variation $u_{N}^{r}(t)$ of type (4.9) with $r:=\sum_{j=1}^{p} m_{j}$ and a vector quantity of order $o\left(h_{N}\right)$ such that

$$
\Delta_{N}\left(p, m_{j}\right)=\Delta^{r} x_{N}\left(t_{1}\right)+o\left(h_{N}\right) \text { as } N \rightarrow \infty
$$

for the corresponding endpoint trajectory increments.

To proceed further, we recall the forms of the cost and inequality constraint functions given in assumption in (b) of Theorem 3.1 and on this basis introduce the following vector mappings from $\mathbb{R}^{n}$ to $R^{l+1}$ defined by

$$
\begin{gathered}
\Phi(x):=\left(\varphi_{0}(x), \ldots, \varphi_{l}(x)\right), \quad \Psi(x):=\left(\psi_{0}(x), \ldots, \psi_{l}(x)\right), \quad \Upsilon(x):=\left(\vartheta_{0}(x), \ldots, \vartheta_{l}(x)\right), \\
L(x):=\Psi(\bar{x})+\Psi^{\prime}(\bar{x})(x-\bar{x})+\Upsilon(x) .
\end{gathered}
$$

It is obvious that all the components $L_{i}$ of the mapping $L$ from (4.11) are convex and represented as sums of convex and linear functions. Thus

$$
L_{i}(\bar{x})=\varphi_{i}(\bar{x}) \text { and } \partial L_{i}(x)=\nabla \psi_{i}(\bar{x})+\partial \vartheta_{i}(\bar{x}) \text { for all } i=0, \ldots, l
$$

by the subdifferential sum rule mentioned in Section 2 .

The next lemma presents an asymptotic consequence of optimality in $\left(P_{N}\right)$ by using multineedle variations of optimal controls.

Lemma 4.4 (asymptotic consequence of optimality). Fix a natural number $r$ independent of $N$ and consider a sequence $\left\{\Delta^{r} x_{N}\left(t_{1}\right)\right\}, N \in \mathbb{N}$, of endpoint trajectory increments generated by r-needle variations of optimal controls. Then there exists an index $i_{0} \in\{0, \ldots, l\}$ independent of $N$ such that

$$
\liminf _{h_{N} \rightarrow 0} \frac{L_{i_{0}}\left(\bar{x}+\Delta^{r} x_{N}\left(t_{1}\right)\right)-L_{i_{0}}(\bar{x})}{h_{N}} \geq 0
$$

for the corresponding component of the mapping L defined in (4.11).

Proof. It follows from the optimality of the sequence $\left\{\bar{x}_{N}\right\}$ that there is an index $i_{0}=i_{0}(N) \in\{0, \ldots, l\}$ such that

$$
\varphi_{i_{0}}\left(\bar{x}_{N}\left(t_{1}\right)+\Delta^{r} x_{N}\left(t_{1}\right)\right) \geq \varphi_{i_{0}}\left(\bar{x}_{N}\left(t_{1}\right)\right) .
$$

Indeed, the inequalities $\varphi_{i}\left(\bar{x}_{N}\left(t_{1}\right)+\Delta^{r} x_{N}\left(t_{1}\right)\right)<\varphi_{i}\left(\bar{x}_{N}\left(t_{1}\right)\right.$, for all $i=0, \ldots, l$ contradict optimality of $\bar{x}_{N}$. $)$ By selecting a subsequence of $\{N\}$ if necessary, we can assume that $i_{0}$ does not depend on $N$ and that $\bar{x}_{N}\left(t_{1}\right) \rightarrow \bar{x}$. Further, it follows from (4.13) by the strict differentiability of $\psi$ at $\bar{x}$ that

$$
\begin{aligned}
& \vartheta_{i_{0}}\left(\bar{x}_{N}\left(t_{1}\right)+\Delta^{r} x_{N}\left(t_{1}\right)\right)-\vartheta_{i_{0}}\left(\bar{x}_{N}\left(t_{1}\right)\right) \geq-\left(\psi_{i_{0}}\left(\bar{x}_{N}\left(t_{1}\right)+\Delta^{r} x_{N}\left(t_{1}\right)\right)-\psi_{i_{0}}\left(\bar{x}_{N}\left(t_{1}\right)\right)\right) \\
& =-\left\langle\nabla \psi_{i_{0}}(\bar{x}), \Delta^{r} x_{N}\left(t_{1}\right)\right\rangle+\widetilde{c}_{N},
\end{aligned}
$$


where $\widetilde{c}_{N}=o\left(\left\|\Delta^{r} x_{N}\left(t_{1}\right)\right\|\right)=o\left(h_{N}\right)$ due to $\left\|\Delta^{r} x_{N}\left(t_{1}\right)\right\|=O\left(h_{N}\right)$ as shown in the proof of [4], Lemma 6.62. We conclude from the relationships above that

$$
\begin{aligned}
& \vartheta_{i_{0}}\left(\bar{x}_{N}\left(t_{1}\right)+\Delta^{r} x_{N}\left(t_{1}\right)\right)+\psi_{i_{0}}(\bar{x})+\left\langle\nabla \psi_{i_{0}}(\bar{x}), \bar{x}_{N}\left(t_{1}\right)+\Delta^{r} x_{N}\left(t_{1}\right)-\bar{x}\right\rangle \\
& \geq \vartheta_{i_{0}}\left(\bar{x}_{N}\left(t_{1}\right)\right)+\psi_{i_{0}}(\bar{x})+\left\langle\nabla \psi_{i_{0}}(\bar{x}), \bar{x}_{N}\left(t_{1}\right)-\bar{x}\right\rangle+\widetilde{c}_{N}
\end{aligned}
$$

which can be written, in the notation of (4.11), as

$$
L_{i_{0}}\left(\bar{x}_{N}\left(t_{1}\right)+\Delta^{r} x_{N}\left(t_{1}\right)\right) \geq L_{i_{0}}\left(\bar{x}_{N}\left(t_{1}\right)\right)+\widetilde{c}_{N} .
$$

Applying now Lemma 4.2, we conclude that there exists a sequence $\left\{c_{N}\right\} \subset \mathbb{R}$ of order $c_{N}=o\left(h_{N}\right)$ as $N \rightarrow \infty$ such that

$$
L_{i_{0}}\left(\bar{x}+\Delta^{r} x_{N}\left(t_{1}\right)\right)-L_{i_{0}}(\bar{x}) \geq c_{N},
$$

which implies in turn that

$$
\liminf _{h_{N} \rightarrow 0} h_{N}^{-1}\left(L_{i_{0}}\left(\bar{x}+\Delta^{r} x_{N}\left(t_{1}\right)\right)-L_{i_{0}}(\bar{x})\right) \geq 0
$$

and thus completes the proof of this lemma.

Next we form the set $E$ by

$$
E:=\left\{\left(x, \nu_{0}, \ldots, \nu_{l}\right) \in \mathbb{R}^{n+l+1} \mid \psi_{i}(\bar{x})+\left\langle\nabla \psi_{i}(\bar{x}), x-\bar{x}\right\rangle+\vartheta_{i}(x) \leq \nu_{i}, i=0, \ldots, l\right\},
$$

which is a combination of the epigraphs of the components of the mapping $L$ in (4.11). Observe that the set $E$ is convex due to the convexity of the functions $\vartheta_{i}$ as $i=0, \ldots, l$ and also that int $E \neq \emptyset$. Define further the convex hull

$$
\Omega_{N}:=\operatorname{co}\left\{\Delta x_{N}\left(t_{1}\right)\right\}
$$

of the endpoint increments of the optimal trajectory $\bar{x}_{N}$ to $\left(P_{N}\right)$ generated by all single needle variations (4.9) with $r=1$ of the optimal control $\bar{u}_{N}$ for each $N \in \mathbb{N}$. The following crucial lemma asserts that the convex set $\left(\bar{x}+\Omega_{N}, L(\bar{x})\right)$ can be shifted by a vector $c_{N}$ of order $o\left(h_{N}\right)$ so that it does not intersect with the interior of the set $E$. We can treat this result as a sequence of primal optimality conditions for $\left(P_{N}\right)$ as $N \rightarrow \infty$.

Lemma 4.5 (primal optimality conditions for discrete approximations). There exists a sequence $\left\{c_{N}\right\} \subset \mathbb{R}^{l+1}$ of order $o\left(h_{N}\right)$ as $N \rightarrow \infty$ such that

$$
\left(\bar{x}+\Omega_{N}, L(\bar{x})+c_{N}\right) \cap \operatorname{int} E=\emptyset, \quad N \in \mathbb{N} .
$$

Proof. Consider the sequence of real numbers

$$
\sigma_{N}:=\min _{y} \max _{i \in\{0, \ldots, l\}}\left(L_{i}(\bar{x}+y)-L_{i}(\bar{x})\right),
$$

where the minimum is taken over the set of $y \in \Omega_{N}$ with $(\bar{x}+y, L(\bar{x})) \in E$; observe that the minimum is reached since this set is compact and nonempty containing $y=0$. From the construction of $\sigma_{N}$ in (4.18) it follows that for any $y_{N} \in \Omega_{N}$ with $\left(\bar{x}+y_{N}, L(\bar{x})\right) \in E$ there exists an index $i_{1}=i_{1}(N) \in\{0, \ldots, l\}$ such that

$$
\sigma_{N} \leq L_{i_{1}}\left(\bar{x}+y_{N}\right)-L_{i_{1}}(\bar{x}) .
$$

Define $c_{N}:=\left(\sigma_{N}, \ldots, \sigma_{N}\right) \in \mathbb{R}^{l+1}$ and observe that

$$
\left(\bar{x}+y_{N}, L(\bar{x})+c_{N}\right) \notin \operatorname{int} E,
$$

since the contrary would mean that $L_{i}\left(\bar{x}+y_{N}\right)<L_{i}(\bar{x})+\sigma_{N}$ for all $i$ and thus contradict (4.19). It follows from (4.18) with $y=0$ that $\sigma_{N} \leq 0$. The assertion of the lemma will now follow from (4.20) if we show that $\sigma_{N}=o\left(h_{N}\right)$ as $N \rightarrow \infty$. 
Assume to the contrary that $\sigma_{N} \neq o\left(h_{N}\right)$ and find a sequence of $y_{N} \in \Omega_{N}$ and negative constants $\beta_{i}$ as $i=0, \ldots, l$ such that

$$
\liminf _{h_{N} \rightarrow 0} h_{N}^{-1}\left(L_{i}\left(\bar{x}+y_{N}\right)-L_{i}(\bar{x})\right) \leq \beta_{i}<0 .
$$

Recalling the definition (4.16) of the set $\Omega_{N}$ and invoking the Carathéodory Theorem, we represent any element $y_{N} \in \Omega_{N}$ by

$$
y_{N}=\sum_{j=1}^{p} \alpha_{j}(N) \Delta_{j} x_{N}\left(t_{1}\right) \text { with } p=n+1,
$$

where $\Delta_{j} x_{N}\left(t_{1}\right)$ are optimal trajectory increments generated by single needle variations of the optimal control $\bar{u}_{N}$ with the needle variation parameters $\left(\tau_{j}(N), v_{j}(N)\right)$ for each $j=1, \ldots, p$ and $N \in \mathbb{N}$, and where $\alpha_{j}(N) \geq 0$ with $\sum_{j=1}^{p} \alpha_{j}(N)=1$. Therefore

$$
\liminf _{h_{N} \rightarrow 0} h_{N}^{-1}\left(L_{i}\left(\bar{x}+\sum_{j=1}^{p} \alpha_{j}(N) \Delta_{j} x_{N}\right)-L_{i}(\bar{x})\right) \leq \beta_{i}<0, i=0, \ldots, l .
$$

For a given number $\eta>0$ to be specified later, define the quantities

$$
\gamma_{j}(N):=\left[\alpha_{j}(N) / \eta\right], \quad j=1, \ldots, p,
$$

where $[a]$ stands as usual for the integer part of the positive number $a$. Along a subsequence (without relabeling) we have $\gamma_{j}(N) \rightarrow \gamma_{j}^{0}$ as $N \rightarrow \infty$ for $j=1, \ldots, p$. Due to Lemma 4.1, there exist subgradients $\xi_{i} \in \partial L_{i}(\bar{x})$ as $i=0, \ldots, l$ such that

$$
\begin{aligned}
& L_{i}\left(\bar{x}+\sum_{j=1}^{p} \gamma_{j}^{0} \Delta_{j} x_{N}\left(t_{1}\right)\right)-L_{i}(\bar{x})=\left\langle\xi_{i}, \sum_{j=1}^{p} \gamma_{j}(N) \Delta_{j} x_{N}\left(t_{1}\right)\right\rangle+o\left(h_{N}\right) \\
& =\frac{1}{\eta}\left\langle\xi_{i}, \sum_{j=1}^{p} \alpha_{j}(N) \Delta_{j} x_{N}\left(t_{1}\right)\right\rangle+\left\langle\xi_{i}, \sum_{j=1}^{p}\left(\gamma_{j}-\frac{\alpha_{j}(N)}{\eta}\right) \Delta_{j} x_{N}\left(t_{1}\right)\right\rangle+o\left(h_{N}\right)
\end{aligned}
$$

along a subsequence of $N \rightarrow \infty$. Let $M$ and $M_{1}$ be positive constants such that that $\left\|\Delta_{j} x_{N}\left(t_{1}\right)\right\| \leq M h_{N}$ and $\left\|\xi_{i}\right\| \leq M_{1}$ for all $i$ and $j$. Since $\left\langle\xi_{i}, y\right\rangle \leq L_{i}(\bar{x}+y)-L_{i}(\bar{x})$ for all $y$, we get from (4.22) that

$$
\begin{aligned}
L_{i}\left(\bar{x}+\sum_{j=1}^{p} \gamma_{j}^{0} \Delta_{j} x_{N}\left(t_{1}\right)\right)-L_{i}(\bar{x}) & \leq \frac{1}{\eta}\left(L_{i}\left(\bar{x}+\sum_{j=1}^{p} \alpha_{j}(N) \Delta_{j} x_{N}\left(t_{1}\right)\right)-L_{i}(\bar{x})\right) \\
& +p M M_{1} h_{N}+o\left(h_{N}\right) .
\end{aligned}
$$

Now select an index $i_{2} \in\{0, \ldots, l\}$ so that $\left|\beta_{i_{2}}\right|=\max \left\{\left|\beta_{i}\right| \mid 0 \leq i \leq l\right\}$ and set $\eta$ above as

$$
\eta=\frac{\beta_{i_{2}}}{\beta_{i_{2}}-p M M_{1}} \in(0,1)
$$

Then it follows from (4.23) and (4.21) that

$$
\begin{aligned}
& \liminf _{h_{N} \rightarrow 0} h_{N}^{-1}\left(L_{i}\left(\bar{x}+\sum_{j=1}^{p} \gamma_{j}^{0} \Delta_{j} x_{N}\right)-L_{i}(\bar{x})\right) \leq \frac{\beta_{i}}{\eta}+p M M_{1} \\
= & \frac{\beta_{i}\left(\beta_{i_{2}}-p M M_{1}\right)}{\beta_{i_{2}}}+p M M_{1}=\frac{p M M_{1}\left(-\beta_{i}+\beta_{i_{2}}\right)+\beta_{i} \beta_{i_{2}}}{\beta_{i_{2}}} \leq \beta_{i}<0
\end{aligned}
$$


for all $i=1, \ldots, l$. Thus inequality (4.21) for convex combinations of $\Delta_{j} x_{N}$ as $j=1, \ldots, p$ implies the same type of inequality (4.24) for their integer combinations. Due to Lemma 4.3 there exists an $r$-needle variation of the optimal control $\bar{u}_{N}$ with $r=\sum_{j=1}^{p} \gamma_{j}^{0}$ such that

$$
\sum_{j=1}^{p} \gamma_{j}^{0} \Delta_{j} x_{N}\left(t_{1}\right)=\Delta^{r} x_{N}\left(t_{1}\right)+o\left(h_{N}\right),
$$

which ensures by (4.24) the inequalities

$$
\liminf _{h_{N} \rightarrow 0} h_{N}^{-1}\left(L_{i}\left(\bar{x}+\Delta^{r} x_{N}\left(t_{1}\right)\right)-L_{i}(\bar{x})\right) \leq \beta_{i}<0 \text { for all } i=0, \ldots, l .
$$

The latter inequalities contradict (4.12) and thus complete the proof of the lemma.

The next lemma based on convex separation transforms the primal optimality conditions (4.17) of Lemma 4.5 into dual conditions via appropriate Lagrange multipliers.

Lemma 4.6 (Lagrange multipliers for discrete approximation problems). Given a limiting point $\bar{x}$ for the endpoint sequence $\left\{\bar{x}_{N}\left(t_{1}\right)\right\}$ of optimal trajectories to $\left(P_{N}\right)$, there exist sequences of Lagrange multipliers $\lambda_{N}=$ $\left(\lambda_{0 N}, \ldots, \lambda_{l N}\right) \in \mathbb{R}^{l+1}$ and dual vectors $\xi_{N} \in \mathbb{R}^{n}$ satisfying the conditions

$$
\left\{\begin{array}{l}
\lambda_{i N} \geq 0 \text { for } i=0, \ldots, l, \quad \sum_{i=0}^{l} \lambda_{i N}^{2}=1, \\
\lambda_{i N} \varphi_{i}(\bar{x})=0 \text { for } i=1, \ldots, l, \quad \text { and } \\
\xi_{N} \in \sum_{i=0}^{l} \lambda_{i N} \partial L_{i}(\bar{x})=\sum_{i=0}^{l} \lambda_{i N}\left(\nabla \psi_{i}(\bar{x})+\partial \vartheta_{i}(\bar{x})\right) .
\end{array}\right.
$$

Furthermore, for an arbitrary sequence of endpoint increments $\Delta x_{N}\left(t_{1}\right)$ generated by single needle variations of optimal controls we have

$$
\left\langle\xi_{N}, \Delta x_{N}\left(t_{1}\right)\right\rangle \leq o\left(h_{N}\right) \text { as } N \rightarrow \infty .
$$

Proof. Applying the classical Separation Theorem to the convex sets $\left(\bar{x}+\Omega_{N}, L(\bar{x})+c_{N}\right)$ and $E$ with empty intersection (4.17), we find dual elements $\widetilde{\lambda}_{N}=\left(\widetilde{\lambda}_{0 N}, \ldots, \widetilde{\lambda}_{l N}\right) \in \mathbb{R}^{l+1}$ and $\widetilde{\xi}_{N} \in \mathbb{R}^{n}$ such that

$$
\left\|\widetilde{\xi}_{N}\right\|^{2}+\sum_{i=0}^{l} \widetilde{\lambda}_{i N}^{2}=1
$$

and that for any $\Delta x_{N}\left(t_{1}\right) \in \Omega_{N}$ and $(x, y) \in E$ the inequality

$$
\left\langle\widetilde{\xi}_{N}, \bar{x}+\Delta x_{N}\left(t_{1}\right)\right\rangle+\left\langle\widetilde{\lambda}_{N}, L(\bar{x})+c_{N}\right\rangle \leq\left\langle\widetilde{\xi}_{N}, x\right\rangle+\left\langle\widetilde{\lambda}_{N}, y\right\rangle
$$

holds, which can be equivalently written as

$$
\left\langle\widetilde{\xi}_{N}, \Delta x_{N}\left(t_{1}\right)\right\rangle+\left\langle\widetilde{\lambda}_{N}, c_{N}\right\rangle \leq\left\langle\widetilde{\xi}_{N}, x-\bar{x}\right\rangle+\left\langle\widetilde{\lambda}_{N}, y-L(\bar{x})\right\rangle .
$$

Setting $\Delta x_{N}\left(t_{1}\right)=0$ in $(4.27)$ gives us

$$
\left\langle\widetilde{\xi}_{N}, x-\bar{x}\right\rangle+\left\langle\widetilde{\lambda}_{N}, y-L(\bar{x})\right\rangle \geq\left\langle\widetilde{\lambda}_{N}, c_{N}\right\rangle,
$$

while by setting $x=\bar{x}$ and $y=L(\bar{x})$ in (4.27) we obtain

$$
\left\langle\widetilde{\xi}_{N}, \Delta x_{N}\left(t_{1}\right)\right\rangle \leq-\left\langle\widetilde{\lambda}_{N}, c_{N}\right\rangle=o\left(h_{N}\right) \text { for any } \Delta x_{N}\left(t_{1}\right) \in \Omega_{N} .
$$


Further, fix $N \in \mathbb{N}$ and denote by $\left(\xi_{N}, \lambda_{N}\right)=\left(\xi_{N}, \lambda_{0 N}, \ldots, \lambda_{l N}\right) \in \mathcal{N}((\bar{x}, L(\bar{x})) ; E)$ the (unique) element of the normal cone $(2.4)$ to the convex set $(4.15)$ at the $(\bar{x}, L(\bar{x}))$ that is nearest point to $\left(\widetilde{\xi}_{N}, \widetilde{\lambda}_{N}\right)$. We conclude from (2.4) and (4.28) that

$$
\left\|\left(\xi_{N}, \lambda_{N}\right)-\left(\widetilde{\xi}_{N}, \widetilde{\lambda}_{N}\right)\right\| \rightarrow 0 \quad \text { as } N \rightarrow \infty
$$

which ensures therefore that

$$
\left\|\xi_{N}\right\|^{2}+\sum_{i=0}^{l} \lambda_{i N}^{2} \rightarrow 1 \quad \text { as } N \rightarrow \infty .
$$

It easily follows from the structure of the set $E$ in (4.15) and of the normal cone to it at the limiting point $(\bar{x}, L(\bar{x}))$ that

$$
\begin{gathered}
\lambda_{i N} \geq 0 \text { for } i=0, \ldots, l, \quad \lambda_{i N} L_{i}(\bar{x})=\lambda_{i N} \varphi_{i}(\bar{x})=0 \text { for } i=1, \ldots, l, \text { and } \\
\xi_{N} \in \sum_{i=0}^{l} \lambda_{i N} \partial L_{i}(\bar{x})=\sum_{i=0}^{l} \lambda_{i N}\left(\nabla \psi_{i}(\bar{x})+\partial \vartheta_{i}(\bar{x})\right) .
\end{gathered}
$$

Taking the last inclusion into account and using (4.31), we renormalize the multipliers $\lambda_{i N}, i=0, \ldots, l$, to satisfy $\sum_{i=0}^{l} \lambda_{i N}^{2}=1$, and thus arrive at all the conditions in (4.25). Finally, it follows from (4.29) and (4.30) that the limiting relationship (4.26) also holds, which thus concludes the proof of the lemma.

To complete the proof of Theorem 3.1, it remains to show that the obtained estimate (4.26) implies the approximate maximum condition (1.7), along the adjoint trajectory $p_{N}(t)$ of system (1.8) satisfying the endpoint/transversality condition

$$
-p_{N}\left(t_{1}\right) \in \sum_{i=0}^{l} \lambda_{i N}\left(\nabla \psi_{i}(\bar{x})+\partial \vartheta_{i}(\bar{x})\right) .
$$

Completing the proof of Theorem 3.1. Employing Lemma 4.6, we find vectors $\xi_{N} \in \mathbb{R}^{n}$ and $\lambda_{N}=$ $\left(\lambda_{0 N}, \ldots, \lambda_{l N}\right) \in \mathbb{R}^{l+1}$ that satisfy all the relationships in (4.25) and (4.26) for every $N \in \mathbb{N}$. Define now $p_{N}\left(t_{1}\right):=-\xi_{N}$ and observe that conditions (4.25) reduce to those in (3.1)-(3.3) of Theorem 3.1 for problems $\left(P_{N}\right)$ with $q=0$, while condition (4.26) can be rewritten in the form

$$
\left\langle p_{N}\left(t_{1}\right), \Delta x_{N}\left(t_{1}\right)\right\rangle \geq o\left(h_{N}\right) .
$$

Recall that $\Delta x_{N}(t)$ are optimal trajectory increments generated by the following single needle variations of optimal control $\bar{u}_{N}(t)$ :

$$
u_{N}(t)= \begin{cases}v(N), & t=\tau(N), \\ \bar{u}_{N}(t), & t \in T_{N}, t \neq \tau(N) .\end{cases}
$$

For any function $p_{N}(t), t \in T_{N} \cup\left\{t_{1}\right\}$, we have the identity

$$
\begin{aligned}
\left\langle p_{N}\left(t_{1}\right), \Delta x_{N}\left(t_{1}\right)\right\rangle= & \sum_{t=t_{0}}^{t_{1}-h_{N}}\left\langle p_{N}\left(t+h_{N}\right)-p_{N}(t), \Delta x_{N}(t)\right\rangle \\
& +\sum_{t=t_{0}}^{t_{1}-h_{N}}\left\langle p_{N}\left(t+h_{N}\right), \Delta x_{N}\left(t+h_{N}\right)-\Delta x_{N}(t)\right\rangle .
\end{aligned}
$$

Using further the notation

$$
\begin{aligned}
& \Delta f(t):=f\left(x_{N}(t), u_{N}(t), t\right)-f\left(\bar{x}_{N}(t), \bar{u}_{N}(t), t\right), \\
& \Delta_{u} f(t):=f\left(\bar{x}_{N}(t), u_{N}(t), t\right)-f\left(\bar{x}_{N}(t), \bar{u}_{N}(t), t\right), \\
& \Delta_{u} H(t):=\left\langle p_{N}\left(t+h_{N}\right), \Delta_{u} f(t)\right\rangle
\end{aligned}
$$


we obtain the representation

$$
\Delta x_{N}\left(t+h_{N}\right)-\Delta x_{N}(t)=h_{N} \Delta f(t)=h_{N}\left[\Delta_{u} f(t)+\frac{\partial f}{\partial x}\left(\bar{x}_{N}(t), \bar{u}_{N}(t), t\right) \Delta x_{N}(t)+\eta_{N}(t)\right],
$$

where the remainder $\eta_{N}(t)$ is defined by

$$
\eta_{N}(t):=\left(\frac{\partial f}{\partial x}\left(\bar{x}_{N}(t), u_{N}(t), t\right)-\frac{\partial f}{\partial x}\left(\bar{x}_{N}(t), \bar{u}_{N}(t), t\right)\right) \Delta x_{N}(t)+o\left(\left\|\Delta x_{N}(t)\right\|\right) .
$$

Since $\Delta x_{N}(t)=0$ for $t \leq \tau=\tau(N)$ and $\left(\frac{\partial f}{\partial x}\left(\bar{x}_{N}(t), u_{N}(t), t\right)-\frac{\partial f}{\partial x}\left(\bar{x}_{N}(t), \bar{u}_{N}(t), t\right)\right)=0$ for all $t \in T_{N}$ except $t=\tau$, the first term in the formula for $\eta_{N}(t)$ vanishes, and therefore we arrive at the relationships $\eta_{N}(t)=$ $o\left(\left\|\Delta x_{N}(t)\right\|\right)=o\left(h_{N}\right)$.

This allows us to represent the second sum on the right-hand side of (4.34) as

$$
\begin{aligned}
\sum_{t=t_{0}}^{t_{1}-h_{N}}\left\langle p_{N}\left(t+h_{N}\right), \Delta\right. & \left.x_{N}\left(t+h_{N}\right)-\Delta x_{N}(t)\right\rangle=h_{N} \sum_{t=t_{0}}^{t_{1}-h_{N}} \Delta_{u} H(t) \\
& +h_{N} \sum_{t=t_{0}}^{t_{1}-h_{N}}\left\langle p_{N}\left(t+h_{N}\right), \frac{\partial f}{\partial x}\left(\bar{x}_{N}(t), \bar{u}_{N}(t), t\right) \Delta x_{N}(t)+\eta_{N}(t)\right\rangle \\
& =h_{N} \Delta_{u} H(\tau)+h_{N} \sum_{t=t_{0}}^{t_{1}-h_{N}}\left\langle p_{N}\left(t+h_{N}\right), \frac{\partial f}{\partial x}\left(\bar{x}_{N}(t), \bar{u}_{N}(t), t\right) \Delta x_{N}(t)\right\rangle+o\left(h_{N}\right) .
\end{aligned}
$$

Taking into account that $p_{N}(t)$ satisfies the adjoint system (1.8), we rewrite the first sum on the right-hand side of $(4.34)$ as

$$
\begin{aligned}
\sum_{t=t_{0}}^{t_{1}-h_{N}}\left\langle p_{N}\left(t+h_{N}\right)-p_{N}(t), \Delta x_{N}(t)\right\rangle & =-h_{N} \sum_{t=t_{0}}^{t_{1}-h_{N}}\left\langle\frac{\partial H}{\partial x}\left(p_{N}\left(t+h_{N}\right) \bar{x}_{N}(t), \bar{u}_{N}(t), t\right), \Delta x_{N}(t)\right\rangle \\
& =-h_{N} \sum_{t=t_{0}}^{t_{1}-h_{N}}\left\langle p_{N}\left(t+h_{N}\right), \frac{\partial f}{\partial x}\left(\bar{x}_{N}(t), \bar{u}_{N}(t), t\right) \Delta x_{N}(t)\right\rangle .
\end{aligned}
$$

It follows from (4.33)-(4.35) that

$$
o\left(h_{N}\right) \leq\left\langle p_{N}\left(t_{1}\right), \Delta x_{N}\left(t_{1}\right)\right\rangle=-h_{N} \Delta_{u} H(\tau)+o\left(h_{N}\right),
$$

which can be written in the form

$$
h_{N} \Delta_{u} H(\tau) \leq o\left(h_{N}\right)
$$

This clearly implies the approximate maximum condition (1.7) at the point $t=\tau(N)$. Since $\tau(N)$ was chosen arbitrarily, we justify the approximate maximum condition for all $t \in T_{N}$ and thus complete the proof of the theorem.

\section{Problems with equality constraints}

In the concluding section of the paper we show that the Approximate Maximum Principle of Theorem 3.1 does not hold if $\delta=0$ in problems $\left(P_{N}\right)$, i.e., in the case of unrelaxed equality constraints. The following simple example illustrates this phenomenon. 
Example 5.1 (violation of the AMP in discrete approximation problems with unrelaxed equality constraints). Consider a two-dimensional continuous-time problem

$$
(P)\left\{\begin{array}{l}
\text { minimize } x(1) \text { subject to } \\
\dot{x}=u \text { a.e. } t \in T:=[0,1], \\
\dot{y}=v \text { a.e. } t \in T, \\
x(0)=y(0)=0, \\
(u(t), v(t)) \in U:=\{(0,0),(-1, \sqrt{2}),(-1,-1)\} \text { a.e. } t \in T, \\
y(1)=0 .
\end{array}\right.
$$

The corresponding discrete approximation problems $\left(P_{N}\right)$ defined in Section 1 with $\delta=0$ are given by

$$
\left(P_{N}\right)\left\{\begin{array}{l}
\text { minimize } x_{N}(1) \text { subject to } \\
x_{N}\left(t+h_{N}\right)=x_{N}(t)+h_{N} u_{N}(t), t \in T_{N}:=\left\{0, h_{N}, \ldots, 1-h_{N}\right\} \\
y_{N}\left(t+h_{N}\right)=y_{N}(t)+h_{N} v_{N}(t), t \in T_{N} \\
x_{N}(0)=y_{N}(0)=0 \\
\left(u_{N}(t), v_{N}(t)\right) \in U, t \in T_{N} \\
y_{N}(1)=0 \\
h_{N}=\frac{1}{N}, N=1,2, \ldots
\end{array}\right.
$$

It is easy to see that an optimal control to problem $(P)$ is

$$
(\bar{u}(t), \bar{v}(t))= \begin{cases}(-1, \sqrt{2}) & \text { for } 0 \leq t \leq 1 /(1+\sqrt{2}), \\ (-1,-1) & \text { for } 1 /(1+\sqrt{2})<t \leq 1 .\end{cases}
$$

We can also observe that, due to the incommensurability of the radical $\sqrt{2}$ and the discretization step $h_{N}=1 / N$, the only feasible control for each discrete approximation problem $\left(P_{N}\right)$ satisfying the constraint $y_{N}(1)=0$ is $\left(u_{N}(t), v_{N}(t)\right) \equiv(0,0)$. It remains to show that this control does not satisfy the necessary optimality conditions of Theorem 3.1.

To proceed, note that the Hamilton-Pontryagin function (1.3) reduces in the this case to

$$
H\left(p_{1 N}, p_{2 N}, u_{N}, v_{N}\right)=p_{1 N} u_{N}+p_{2 N} v_{N} \text { for all } t \in T_{N},
$$

where both $p_{1 N}$ and $p_{2 N}$ are constants due to the adjoint system (1.6). The endpoint condition (3.3) is written now as

$$
\left(p_{1 N}(1), p_{2 N}(1)\right)=-\left.\nabla_{(x, y)}\left(\lambda_{0 N} x+\lambda_{1 N} y\right)\right|_{(x, y)=\left(x_{N}(1), y_{N}(1)\right)}=\left(-\lambda_{0 N},-\lambda_{1 N}\right)
$$

with $\left(\lambda_{0, N}, \lambda_{1 N}\right)$ satisfying the sign and nontriviality conditions

$$
\lambda_{0 N} \geq 0 \text { and } \lambda_{0 N}^{2}+\lambda_{1 N}^{2}=1
$$

by (3.1). This allows us to rewrite function (5.1) in the form

$$
H\left(\lambda_{0 N}, \lambda_{1 N}, u_{N}, v_{N}\right)=-\lambda_{0 N} u_{N}-\lambda_{1 N} v_{N} .
$$

A simple analysis shows that for any $\lambda_{0 N}$ and $\lambda_{1 N}$ satisfying (5.2) there exists a constant $\alpha>0$ such that, depending on the sign of $\lambda_{1 N}$, we have

$$
\left\{\begin{array}{l}
\text { either } H\left(\lambda_{0 N}, \lambda_{1 N},-1, \sqrt{2}\right)>H\left(\lambda_{0 N}, \lambda_{1 N}, 0,0\right)+\alpha \\
\text { or } H\left(\lambda_{0 N}, \lambda_{1 N},-1,-1\right)>H\left(\lambda_{0 N}, \lambda_{1 N}, 0,0\right)+\alpha,
\end{array}\right.
$$

which contradicts the approximate maximum condition (1.7).

Note finally that this example can be slightly modified to illustrate the failure of the AMP if the equality constraint $y_{N}(1)=0$ in problems $\left(P_{N}\right)$ of Example 5.1 is relaxed to $\left|y_{N}(1)\right| \leq \delta_{N}$, where $\delta_{N} \downarrow 0$ too fast as $N \rightarrow \infty$; cf. the discussions in Remark 3.2. 


\section{REFERENCES}

[1] R. Gabasov and F.M. Kirillova, On the extension of the maximum principle by L.S. Pontryagin to discrete systems. Autom. Remote Control 27 (1966) 1878-1882.

[2] B.S. Mordukhovich, Approximate maximum principle fot finite-difference control systems. Comput. Maths. Math. Phys. 28 (1988) 106-114.

[3] B.S. Mordukhovich, Discrete approximations and refined Euler-Lagrange conditions for nonconvex differential inclusions. SIAM J. Control Optim. 33 (1995) 882-915.

[4] B.S. Mordukhovich, Variational Analysis and Generalized Differentiation, I: Basic Theory, II: Applications. Springer, Berlin (2006).

[5] B.S. Mordukhovich and I. Shvartsman, The approximate maximum principle in constrained optimal control. SIAM J. Control Optim. 43 (2004) 1037-1062.

[6] B.S. Mordukhovich and I. Shvartsman, Nonsmooth approximate maximum principle in optimal control. Proc. 50th IEEE Conf. Dec. Cont. Orlando, FL (2011).

[7] K. Nitka-Styczen, Approximate discrete maximum principle for the discrete approximation of optimal periodic control problems, Int. J. Control 50 (1989) 1863-1871.

[8] L.C. Pontryagin, V.G. Boltyanskii, R.V. Gamkrelidze and E.F. Mishchenko, The Mathematical Theory of Optimal Processes. Wiley, New York (1962).

[9] R.T. Rockafellar, Convex Analysis. Princeton University Press, Princeton, NJ (1973).

[10] G.V. Smirnov, Introduction to the Theory of Differential Inclusions. American Mathematical Society, Providence, RI (2002).

[11] R.B. Vinter, Optimal Control. Birkhäuser, Boston (2000). 